\title{
ARTICLE
}

\section{Gynura Rhizoma containing pyrrolizidine alkaloids induces the hepatic sinusoidal obstruction syndrome in mice via upregulating fibrosis-related factors}

\author{
Fang Zhang ${ }^{1,2}$, Yue Zhou ${ }^{1}$, Xiao Yang ${ }^{1}$, Ai-zhen Xiong ${ }^{1}$, Zheng-tao Wang ${ }^{1}$ and Li Yang ${ }^{1,2}$
}

Recently, hepatic sinusoidal obstruction syndrome (HSOS) caused by herbal preparations containing pyrrolizidine alkaloids (PAs), such as Gynura Rhizoma (Tusanqi), has gained global attention. However, the lack of a reliable and reproducible animal model has greatly hampered mechanistic studies. Therefore, we aimed to establish a reproducible HSOS mouse model and investigate the hepatotoxic mechanism. The model was established by intragastrical administration of Gynura Rhizoma extract, i.e., $1.0 \mathrm{~g}$ extract/kg per day (equal to $16.7 \mathrm{~g}$ crude drug/kg per day based on extraction rate and $49.1 \mathrm{mg}$ PA/kg per day based on the total PA content in the extract determined) for 40 successive days. Then, the mice were sacrificed, and their blood samples and livers were collected for analyses. Using hematoxylin-eosin (HE) and Masson staining, scanning electron microscopy imaging, clinical biomarkers, and other assays, we showed that the HSOS was successfully induced in our mouse model. Furthermore, we detected the key factors involved in liver fibrosis in the mice, revealing significantly increased hydroxyproline concentration; elevated expression of a-smooth muscle actin (a-SMA) and fibrosis-related genes such as Collagen-1, Collagen-3, Mmp2, Mmp13, Timp1, Timp3, and Activin, upregulated Smad3 phosphorylation, and increased serum TGF- $\beta$ levels. Moreover, pro-inflammatory cytokines, including Tnf- $a$, II-1 $\beta$, and II- 6 , were also increased in the model. All these results demonstrate the key roles of the TGF- $\beta$-Smad 3 and inflammatory signaling pathways in this Gynura Rhizoma-induced HSOS mouse model, suggesting that blockade of fibrosis and/or inflammation should be an effective treatment for HSOS.

Keywords: hepatic sinusoidal obstruction syndrome; herbal-induced liver injury; pyrrolizidine alkaloid; Gynura Rhizoma; liver fibrosis; inflammation

Acta Pharmacologica Sinica (2019) 40:781-789; https://doi.org/10.1038/s41401-018-0155-y

\section{INTRODUCTION}

Herbal products are distributed as herbal medicines or dietary supplements in many countries. However, herbal drug-induced liver injury (HILI) has been reported in many countries around the world recently [1-3]. Over 60 herbal medicines can cause HILI. HILI has gained global attention and is now a concerning problem in traditional herbal medicine. Among the HILI cases are hundreds of reports of hepatic sinusoidal obstruction syndrome (HSOS) caused by herbal products containing pyrrolizidine alkaloids (PAs) or foods contaminated with PAs [3-6]. Therefore, numerous countries and organizations, such as the World Health Organization, the Food Agriculture Organization of the United Nations, the German Federal Health Bureau, and the State Pharmacopoeia Commission of the China, have enacted guidelines on the prevention of PA exposure. However, the cases of HSOS induced by PAs have still markedly increased since the end of the past century, especially by self-medication with Gynura Rhizoma (Tusanqi) $[1,2,6]$.

Gynura Rhizoma is the dried root of Gynura japonica (Thunb.) Juel. (syn. Gynura segetum (Lour.) Merr.), which grows mainly in Afghanistan, China, Ethiopia, Iraq, South Africa, and Uzbekistan. It has been used as a folk herbal medicine for thousands of years because it shows wide clinical effects against various ailments, such as promoting blood circulation and detumescence and relieving pain. However, recent studies show that Gynura Rhizoma can induce severe toxic reactions because it contains a large amount of PAs [7-10]. PAs are among the most hepatotoxic natural compounds $[6,11]$. Over 660 PAs and their $N$-oxides exist in more than 6000 flowering plants globally. Most PAs are toxic due to metabolic activation by cytochrome P450 (CYP450) to form the highly active metabolites, dehydropyrrolizidine alkaloids (DHPAs). DHPAs are chemically reactive electrophilic metabolites with extremely short half-lives. Once formed, DHPAs rapidly interact with cellular macromolecules such as proteins to form 2,3dihydro- $1 \mathrm{H}$-pyrrolizineprotein (pyrrole-protein) adducts, which further promote HSOS [11, 12].

Clinical causality assessment is key to the effective treatment of HSOS. The Roussel Uclaf Causality Assessment Method score system, which includes a combination of novel markers, such as pyrrole-protein adducts and miRNAs, showed some potential for the causality assessment of PA-induced HSOS $[9,13,14]$. However,

\footnotetext{
${ }^{1}$ The Ministry of Education Key Laboratory for Standardization of Chinese Medicines and the SATCM Key Laboratory for New Resources and Quality Evaluation of Chinese Medicines, Institute of Chinese Materia Medica, Shanghai University of Traditional Chinese Medicine, Shanghai 201203 , China and ${ }^{2}$ Institute of Interdisciplinary Integrative Medicine Research, Shanghai University of Traditional Chinese Medicine, Shanghai 201203, China Correspondence: Ai-zhen Xiong (aizhenxiong@shutcm.edu.cn) or Li Yang (yl7@shutcm.edu.cn)
}

Received: 27 February 2018 Accepted: 18 July 2018

Published online: 26 October 2018 
HSOS caused by PAs is rather difficult to confirm quickly. It is now clearly established that the first morphological change in HSOS occurs in the sinusoidal endothelial cells (SECs), which become round and flat, followed by the formation of gaps within the sinusoidal barrier, with subsequent entrance of red blood cells into the space of Disse [15]. These finally lead to the embolization of sinusoidal lining cells and result in hepatic sinusoid obstruction in zone 3 of the hepatic acinus. However, in the intermediate and late stages, the subintimal lesions become fibrotic and eventually lead to perivenular fibrosis, pericellular fibrosis, and worse, cirrhosis [15-17]. Given the high mortality rate (over $80 \%$ ) of severe HSOS, great efforts have been made to illustrate the underlying mechanism for its effective therapy. However, there is still no effective drug for the treatment of HSOS, except defibrotide, which was recently approved in Europe for the treatment of severe HSOS secondary to hematopoietic stem cell transplantation [18]. In addition, there is no effective treatment for PA-induced HSOS other than some natural compounds [19-21] (i.e., quercetin, baicalein, chlorogenic acid) or synthesized chemicals [22] (i.e., prednisone) that have shown protective effects against PA-induced HSOS in rodents. In view of this, more efforts are needed to illustrate the underlying mechanism of HSOS for its effective therapy.

Therefore, the present study was designed to establish a reliable and reproducible HSOS mouse model to discover the mechanism of HSOS. As previously reported, HSOS begins with endothelial cell damage with microthromboses and fibrin deposition and causes severe fibrosis. However, limited research has been performed to characterize the impaired fibrosis-related factors in PA-induced HSOS. Therefore, we evaluated molecular markers related to hepatic fibrosis and inflammation in the HSOS mouse model to investigate the underlying hepatotoxic mechanisms.

\section{MATERIALS AND METHODS}

Herbal extracts

Gynura Rhizoma (collected from Yangzhou, Jiangsu, China) was authenticated by the authors and dried at $60^{\circ} \mathrm{C}$ overnight before being powdered. The total extract was prepared for toxicity evaluation following our previously reported procedure [23]: pulverized crude powder $(0.5 \mathrm{~kg})$ was extracted thrice with eight volumes of $95 \%$ ethanol under reflux for $1 \mathrm{~h}$ for each extraction. The extracts were combined and concentrated to dryness to get the total extract. Three batches of total extract were prepared individually, and the extraction rates $(w / w)$ were calculated. The UPLC-MS chemoprofile of the total extracts were performed (Supplementary Text S1 and Fig. S1). And the levels of the main PAs in the extract were measured via a UPLC-MS-selected ion monitoring approach on a Waters ACQUITY UPLC ZQ2000 system (Waters Corporation, Milford, MA, USA) (Supplementary Text S1 and Table S1).

\section{Animal experiments}

Male C57/BL mice (20-22 g, 8 weeks old) were obtained from the Laboratory Animal Center of Shanghai University of Traditional Chinese Medicine (SHUTCM, Shanghai, China). The mice were kept under standard conditions at a temperature of $25 \pm 2{ }^{\circ} \mathrm{C}$, relative humidity of $55 \pm 5 \%$, room air changes $12-18$ times/h, and a 12-h light/dark cycle. Animal welfare and the animal experimental protocols were strictly consistent with the related ethics regulations of SHUTCM. All experiments were approved by the animal research committee of SHUTCM (Registration No. SZY201609007, 20 September 2016). Before treatment, the total extract was suspended in $0.5 \%$ solution of sodium carboxyl methyl cellulose (CMC-Na). The mice were randomly divided into two groups of 12 . The two groups were, respectively, treated with $0.5 \%$ CMC-Na (control group) or $1.0 \mathrm{~g}$ total extract/kg per day (model group; equal to $16.7 \mathrm{~g}$ crude drug/kg per day based on extraction rate and $49.1 \mathrm{mg} P A s / \mathrm{kg}$ per day based on the total PA content in the extract) by intragastric administration on a daily basis for 40 days. Each animal was anesthetized with sodium pentobarbital and sacrificed at $24 \mathrm{~h}$ after the last administration. Blood samples and livers were collected. The blood samples were collected and coagulated for $2 \mathrm{~h}$ at room temperature to separate the sera.

Serum clinical biomarker assays and liver histology

An aliquot of each serum sample was used to examine the clinical biochemistry indexes alanine aminotransferase (ALT) activity, aspartate aminotransferase (AST) activity, and total bilirubin (TBIL) level by using a Hitachi Automatic Analyzer 7080 (Hitachi HighTech Science Systems Corp., Ibaraki, Japan). The remaining samples were stored at $-80^{\circ} \mathrm{C}$ for further assays.

An aliquot of each liver was immersed in formaldehyde and embedded with paraffin. The remaining samples were frozen in liquid nitrogen immediately after collection and stored at $-80^{\circ}$ $C$ for further assays. Paraffin-embedded specimens were sliced into serial sections of $3-5 \mu \mathrm{m}$. Sections were stained with hematoxylin-eosin (HE), Masson trichrome, Sirus red or antibodies for immunohistochemistry (IHC). For IHC assay, sections were deparaffinized and dehydrated before being incubated with anti-MMP9 (ab38898, polyclonal, 1:200 dilution, Abcam plc, Cambridge, UK), anti-p-Smad3 (9520, monoclonal, 1:200 dilution, Cell Signaling Technology, Danvers, MA, USA), anti-a-SMA (ab5694, polyclonal, 1:200 dilution, Abcam plc, Cambridge, UK), and anti-desmin (ab15200, polyclonal, 1:200 dilution, Abcam plc, Cambridge, UK). Six fields of each slide were randomly selected and observed under a Nikon Eclipse 50i microscope (Nikon Corp., Tokyo, Japan).

Three mice in each group were perfused first with saline through the abdominal aorta and then with a fixative solution containing $2.5 \%$ glutaraldehyde. An aliquot of each liver was then cut into small pieces (approximately $1 \mathrm{~mm}^{3}$ ), dehydrated and sputter-coated with gold before being observed under a Hitachi S4700 scanning electron microscope (Hitachi High-Tech Science Systems Corp., Ibaraki, Japan). Six fields were randomly selected and observed for each sample.

\section{RT-PCR assay}

Total liver tissue RNA was extracted by Trizol Reagent according to the manufacturer's instructions (Thermo Fisher, Waltham, MA, USA). In addition, $1 \mu \mathrm{g}$ of the total RNA of each liver was transcribed to cDNA using a PrimeScript RT Master Mix kit (TaKaRa, Shiga, Japan). Gene expression was examined by a SYBR real-time PCR kit (TaKaRa, Shiga, Japan) on an ABI PRISM 7700 sequence detection system (Thermo Fisher, Waltham, MA, USA). The primers used in the study are listed in Table 1 . The relative expression of each target gene was quantified after normalization to $\beta$-actin using densitometry (relative RNA expression $=$ target gene $/ \beta$ actin).

\section{Western blotting analysis}

Approximately $50 \mathrm{mg}$ of each liver was homogenized in radio immunoprecipitation assay lysis buffer (Thermo Fisher, Waltham, MA, USA) with the addition of phosphatase inhibitors and protease inhibitor cocktail (Roche, Mannheim, Germany). After being placed on ice for $30 \mathrm{~min}$, the mixture was centrifuged at $12,000 \mathrm{rpm}$ for $15 \mathrm{~min}$ at $4^{\circ} \mathrm{C}$ to obtain the protein lysate. In addition, the protein concentration was detected by the bicinchoninic acid method (YEASON, Shanghai, China) and normalized before being denatured at $100^{\circ} \mathrm{C}$ for $10 \mathrm{~min}$ with lysis buffer (YEASON, Shanghai, China). Approximately $30 \mu \mathrm{g}$ protein of each sample was then subjected to $10 \%$ sodium dodecyl sulfate-polyacrylamide gel electrophoresis and Western blotting. Specific primary antibodies for a-SMA (42 kDa, ab32575, Abcam plc, Cambridge, UK) and $\beta$-actin ( $43 \mathrm{kDa}, 4970$, Cell 


\begin{tabular}{|c|c|c|}
\hline Primer & $\mathrm{S}$ or AS & Sequence $\left(5^{\prime}-3^{\prime}\right)$ \\
\hline \multirow[t]{2}{*}{ Tnf- $a$} & $S$ & AAGCCTGTAGCCCACGTCGTA \\
\hline & AS & GGCACCACTAGTTGGTTGTCTTTG \\
\hline \multirow[t]{2}{*}{$11-6$} & $S$ & TGGGGCTCTTCAAAAGCTCC \\
\hline & AS & AGGAACTATCACCGGATCTTCAA \\
\hline \multirow[t]{2}{*}{$\|-1 \beta$} & $\mathrm{S}$ & GGTGTGTGACGTTCCCATTAGAC \\
\hline & AS & CATGGAGAATATCACTTGTTGGTTGA \\
\hline \multirow[t]{2}{*}{ Activin } & $\mathrm{S}$ & GGGCCTTTTGATGCTGTCG \\
\hline & AS & TGGCAGAATGGTCTCTTGCAG \\
\hline \multirow[t]{2}{*}{ Follistatin } & $S$ & CAAGCCCAGAAGGTCCTACAG \\
\hline & AS & GACTTGTGCATGTCTCCCCAG \\
\hline \multirow[t]{2}{*}{ Collagen-1 } & $\mathrm{S}$ & GCCCAGAGATAGAGTGACCTG \\
\hline & AS & CGCACTTCTCGAAAGTTGCTG \\
\hline \multirow[t]{2}{*}{ Collagen-3 } & $\mathrm{S}$ & ACATGCTCAACGTGAAGGG \\
\hline & AS & TGCCAACTTGGAAGCAGTCAT \\
\hline \multirow[t]{2}{*}{ Mmp2 } & $\mathrm{S}$ & TGGGGGAGATTCTCACTTTG \\
\hline & AS & CCATCAGCGTTCCCATACTT \\
\hline \multirow[t]{2}{*}{ Mmp9 } & $\mathrm{S}$ & CCACCACAGCCAACTATGACCAG \\
\hline & AS & AAGACGAAGGGGAAGACGCACA \\
\hline \multirow[t]{2}{*}{ Mmp13 } & $\mathrm{S}$ & AGGTTGAGGCTGAGCTCTTT \\
\hline & AS & AAGGTCACGGGATGGATGTT \\
\hline \multirow[t]{2}{*}{ Timp1 } & $\mathrm{S}$ & CAGTGTTTCCCTGTTTATCTATCCC \\
\hline & AS & AGTGACGGCTCTGGTAGTCC \\
\hline \multirow[t]{2}{*}{ Timp2 } & $\mathrm{S}$ & TGGACGTTGGAGGAAAGAAGGAG \\
\hline & AS & TTCTGGGTGATGCTAAGCGTGTC \\
\hline \multirow[t]{2}{*}{ Timp3 } & $\mathrm{S}$ & ACTACTTGCCTTGTTTTGTGA \\
\hline & AS & GCTGATGCTCTTGTCTGG \\
\hline \multirow[t]{2}{*}{ Collagen-la1 } & $\mathrm{S}$ & GCTCCTCTTAGGGGCCACT \\
\hline & AS & CCACGTCTCACCATTGGGG \\
\hline \multirow[t]{2}{*}{ Collagen-la2 } & S & GTAACTTCGTGCCTAGCAACA \\
\hline & AS & CCTTTGTCAGAATACTGAGCAGC \\
\hline \multirow[t]{2}{*}{$\beta$-Actin } & $S$ & GGCTGTATTCCCCTCCATCG \\
\hline & AS & CCAGTTGGTAACAATGCCATGT \\
\hline
\end{tabular}

Signaling Technology, Danvers, MA, USA) were employed together with horseradish peroxidase-conjugated anti-rabbit secondary antibodies. The immune complexes were visualized using the enhanced chemiluminescence method. The blots of the bands were quantified with Tanon FLT Caption (Tanon, Shanghai, China). The relative expression level of a-SMA was calculated using $\beta$ actin as the internal control (relative expression level of $a-S M A=$ a-SMA/ $\beta$-actin).

Enzyme-linked immunosorbent assay (ELISA)

Protein levels of transforming growth factor beta (TGF- $\beta$ ) in serum (YIFAN Biological Technology, Shanghai, China) and hydroxyproline in liver tissue (KEJIAN Biology Science and Technology Co. Ltd., Shanghai, China) were detected by ELISA kits, according to the manufacturers' instructions.

Statistical analysis

Data were analyzed by the paired or unpaired $t$-test and are presented as the mean \pm standard error (SD) $(n=6-12)$. Intergroup differences were considered significant when ${ }^{*} P<0.05$ and highly significant when ${ }^{* *} P<0.01$ or ${ }^{* * *} P<0.001$.

\section{RESULTS}

Chemical standardization of Gynura Rhizoma extract

Three batches of total extract were separately prepared as described [23]. The average extraction rate of Gynura Rhizoma extracts was $6.0 \%(\mathrm{RSD}=4.7 \%, n=3)$. The chemoprofile based on UPLC-MS analysis and the quantification of major hepatotoxic PA (HPA) structures in the extracts were performed to ensure the repeatability of the extracts. As shown in Supplementary Fig. S1, senecionine $\mathrm{N}$-oxide and seneciphylline $\mathrm{N}$-oxide were the main HPAs in the total extracts. Besides, 10 more HPAs structures, including senecionine and seneciphylline, were identified. The total content of the 12 HPAs was $49.1 \mathrm{mg} / \mathrm{g}$ extract $(\mathrm{RSD}=7.1 \%$, $n=3$ ) (Supplementary Table S1). As reported, PA $N$-oxides can be easily reduced to their corresponding parent alkaloids by certain enzymes, resulting in similar toxicity to their corresponding parent alkaloids [24]. The amount of senecionine and its $N$-oxide was 15.9 $\mathrm{mg} / \mathrm{g} \mathrm{TE}$, while the amount of seneciphylline and its $\mathrm{N}$-oxide were $25.9 \mathrm{mg} / \mathrm{g}$ TE. Therefore, the four compounds accounted for $85.2 \%$ of the total HPAs in TE.

Evaluation of an HSOS mouse model caused by multiple administrations of Gynura Rhizoma

In the present study, mice in the model group were intragastrically gavaged with total extract of Gynura Rhizoma ( $1.0 \mathrm{~g}$ total extract/ $\mathrm{kg}$ per day) daily for 40 successive days. The dosage equaled 16.7 $\mathrm{g}$ crude drug/kg per day based on the extraction rate and $49.1 \mathrm{mg}$ $\mathrm{PA} / \mathrm{kg}$ per day based on the total PA content. As reported previously, HSOS patients show significant hepatomegaly, ascites and jaundice, as well as increased ALT, AST, and TBIL values $[25,26]$. From the first day of administration, all mice were closely observed for any abnormality related to these symptoms. Mice in the model group were found to exhibit piloerection in the second week, and some showed decreased movement and distended abdomen in the fourth week, indicating the existence of ascites. When sacrificed, mice in the model group had hemostasis, hepatomegaly, and a dark-red liver (Fig. 1a). HE staining illustrated lobular structure damage, coagulative necrosis of hepatocytes, sinusoid hemorrhage, and endothelial damage to the central vein (Fig. 1b). In addition, Masson staining showed serious hepatic sinusoidal fibrosis (Fig. 1b). Significantly increased serum ALT activity, AST activity, and TBIL levels were also observed in the model group (Fig. 1c). Therefore, the HSOS mice showed similar symptoms to HSOS patients.

Sinusoidal injury happens in the early stage of HSOS. It induces damage to the fenestration of SECs, which forces SECs to transfigure into a disc-like shape. At the same time, matrix metalloproteinase 9 (MMP9) is released to facilitate loss of SECs by the degradation of the extracellular matrix [27, 28]. Scanning electron microscopy images showed dilatation of sinusoids, enlarged and damaged fenestrae, and severe congestion in the liver of the model mouse (Fig. 1d). IHC also indicated increased MMP9-positive cells in the hepatic sinusoid and central vein of the model mouse (Fig. 1e). These results suggested that sinusoidal injury happened in the model group.

These observations show the HSOS mouse model was successfully established in our study. The severity of HSOS was also quantified by scoring four categories of damage, including endothelial damage to the central venules, subendothelial hemorrhage, sinusoidal hemorrhage, and subendothelial fibrosis. Our model had severe HSOS (Table 2).

Hepatic fibrosis in Gynura Rhizoma-induced HSOS mouse model Previous studies have reported that intense hepatic fibrosis happens in the development of HSOS. We next assessed our HSOS mouse model by detecting fibrosis-related molecules. Hydroxyproline is an amino acid unique to collagen in the mammalian body. The excessive accumulation of hydroxyproline can lead to dense fibrosis [29]. As results, hepatic protein level of 

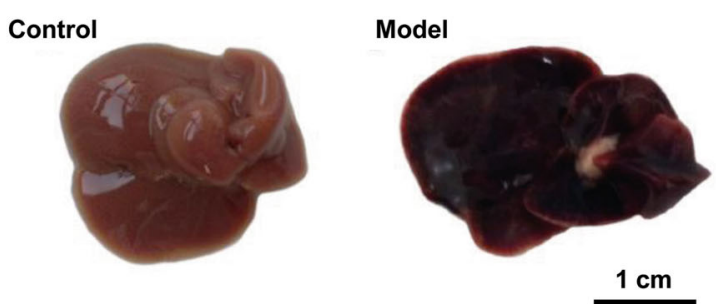

b
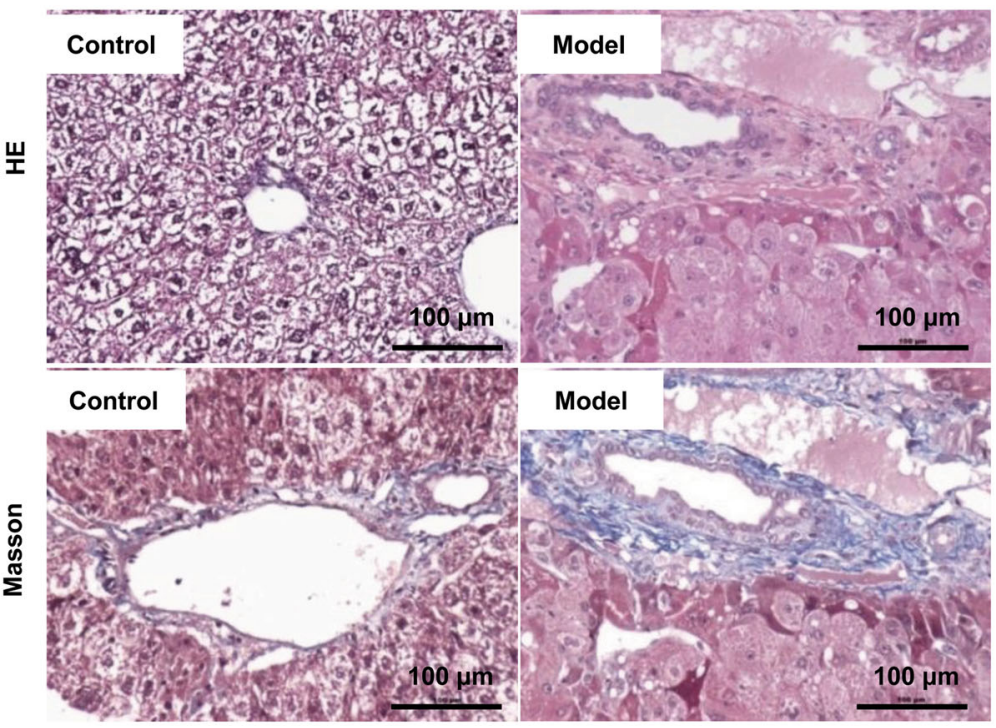

C
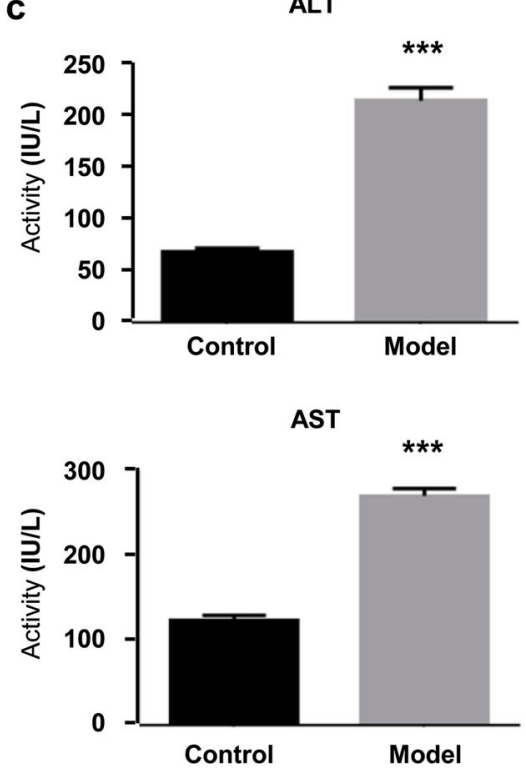

TBIL

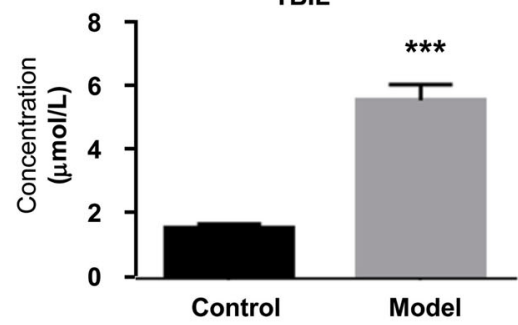

d
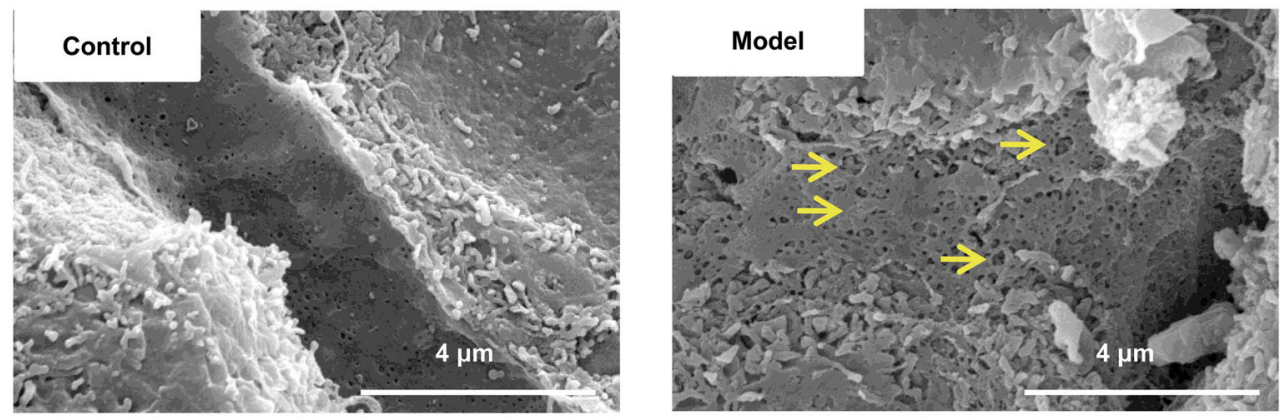

e
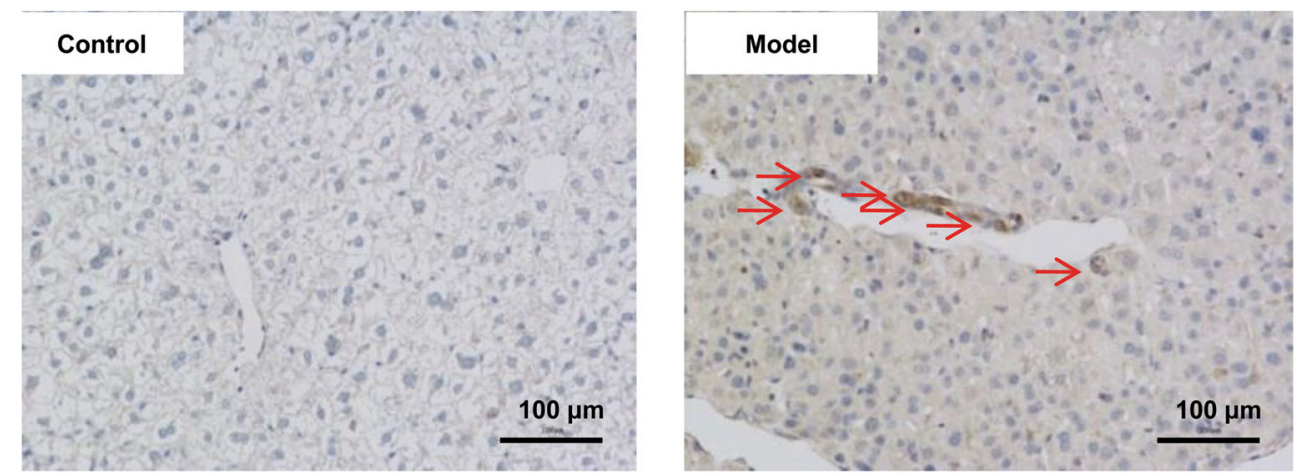

Fig. 1 Evaluation of the HSOS mouse model established by 40 successive days of administration of Gynura Rhizoma, i.e., $1.0 \mathrm{~g}$ total extract/kg per day (equal to $16.7 \mathrm{~g}$ crude drug/kg per day based on the extraction rate and $49.1 \mathrm{mg}$ PAper daykg per day based on the total PA content in the extract). a Images of liver tissues (bar is $1 \mathrm{~cm}$ ). $\mathbf{b} \mathrm{HE}$ and Masson staining of liver tissues (400x magnification, bar is $100 \mu \mathrm{m})$. c Serum ALT and AST activities and TBIL levels. d Scanning electron microscopy images of liver tissues (40,000x magnification, bar is $4 \mu \mathrm{m})$. Yellow arrows indicate dilatation of sinusoids, enlarged and damaged fenestrae, and severe congestion. e MMP9 immunohistochemical staining of liver tissues (400x magnification, bar is $100 \mu \mathrm{m})$. Red arrows indicate MMP9-positive cells in the hepatic sinusoid and central vein. Values are expressed as the mean \pm SD $(n=12)$. Statistics were analyzed by paired $t$-test. ${ }^{* * *} P<0.001$ vs. control group. Six fields were randomly selected and observed for each sample 
Table 2. Evaluation of the severity of the HSOS mouse model induced by Gynura Rhizoma

\begin{tabular}{ll}
\hline Category of damage & Score \\
\hline Endothelial damage of the central venules & $1.2 \pm 0.4^{* *}$ \\
Subendothelial hemorrhage & $1.3 \pm 0.5^{* *}$ \\
Sinusoidal hemorrhage & $1.2 \pm 0.4^{* * *}$ \\
Subendothelial fibrosis & $1.2 \pm 0.4^{* *}$ \\
Total HSOS score & $4.3 \pm 1.0^{* * *}$ \\
\hline Statistics were analyzed by unpaired $t$-test $(n=12){ }^{* * *} P<0.01,{ }^{* * *} P<0.001$ \\
vs. control group
\end{tabular}

hydroxyproline was significantly increased in the model group (Fig. 2a). a-SMA, which is an essential marker of hepatic stellate cell (HSC) activation and directly induces fibrosis [30], was elevated by 1.7 -fold (1.00 in control group vs. 2.73 in model group) in the model group (Fig. 2b). Collagen-positive cells were also increased by $30.3 \%(0.2 \%$ in control group vs. $30.5 \%$ in model group) in the model group (Fig. 2c). Meanwhile, IHC for a-SMA and Sirus red staining of collagen proteins also showed significantly increased a-SMA and collagen-positive cells (Fig. 2d). Consistently, hepatic Collagen-1 (1.00 in control group vs. 2.22 in model group) and Collagen-3 (1.00 in control group vs. 2.61 in model group) were also increased by 1.2-fold and 1.6-fold, respectively, in the model group (Fig. 2e). The hepatic mRNA expression of fibrosisinducing genes, including Mmp2 (1.00 in control group vs. 14.93 in model group), Mmp13 (1.00 in control group vs. 4.09 in model group), Timp1 (1.00 in control group vs. 47.08 in model group), and Timp2 (1.00 in control group vs. 8.10 in model group) were also significantly increased by $3.1-46.1$-fold in the model group (Fig. 2e).

Key role of TGF- $\beta$-Smad3 signal pathway in the HSOS mouse model induced by Gynura Rhizoma

The TGF- $\beta$-Smad3 signal pathway has been reported to play a critical role in fibrosis. Therefore, TGF- $\beta$ secretion in serum was first examined by ELISA. As shown in Fig. 3a, the protein level of TGF- $\beta$ in serum was increased in the model group. The hepatic mRNA expression of other members of the TGF- $\beta$ family, including Activin and Follistatin, was also detected by RT-PCR. Activin was significantly increased by 2.0 -fold (1.00 in control group vs. 3.04 in model group). However, Follistatin, which is a factor that antagonizes Activin, was decreased by $32.0 \%$ (1.00 in control group vs. 0.68 in model group) (Fig. $3 b$ ). In addition, markedly increased phosphorylation of Smad3 was found in the model group (Fig. 3c). Moreover, the mRNA expression of Collagen-la1 was increased by 5.7-fold (1.00 in control group vs. 6.69 in model group) in the model group (Fig. 3d). Therefore, our data indicate that the TGF- $\beta$-Smad3 signal pathway might play an important role in the development of hepatic fibrosis in the Gynura Rhizomainduced HSOS mouse model.

Involvement of proinflammatory cytokines in the HSOS mouse model induced by Gynura Rhizoma

The mRNA expression of Tnf- $a, \|-1 \beta$, and II- 6 in the liver tissues was assessed by RT-PCR. As shown in Fig. 4, Tnf-a-was significantly increased by 3.0 -fold ( 1.00 in control group vs. 3.97 in model group) in the model group. II- $1 \beta$ and II- 6 were also elevated in the model group, indicating that an inflammatory signal was triggered in the Gynura Rhizoma-induced HSOS mouse model.

\section{DISCUSSION}

HSOS, also called hepatic veno-occlusive disease, is a rare disease but has high mortality. The most frequent cause of HSOS is high- dose chemotherapy in recipients of hematopoietic stem cell transplantation in Western Europe and the US [25]. However, exposure to herbal preparations or foods contaminated with PAs is the leading cause of HSOS in developing countries, such as China, Afghanistan, and Ethiopia [25]. It is associated with clinical symptoms of hepatomegaly, ascites, jaundice, and hyperbilirubinemia resulting from fibrous obliteration of small centrilobular hepatic venules $[25,26]$. Several experimentally induced HSOS models show similar pathology to human HSOS patients. Robinson et al. reported a chronic mouse model of oxaliplatininduced HSOS [31], in which mice were treated with intraperitoneal oxaliplatin followed $2 \mathrm{~h}$ later by fluorouracil (5-FU) and folinic acid on a weekly basis for 5 weeks. The model is a successful experimental chronic model and shows similar histological features to those of HSOS patients receiving conversion therapy for colorectal liver metastasis. Therefore, it can be used to explore in detail the pathogenesis of this condition. Defibrotide was recently approved in Europe for the treatment of severe HSOS secondary to hematopoietic stem cell transplantation [18]. Unfortunately, there are no effective drugs for clinical treatment of PA-induced HSOS. In view of this, more direct evidence of the underlying mechanism of HSOS is needed for the development of effective therapies. However, the lack of a reliable and reproducible animal model has greatly hampered the mechanistic study of PA-induced HSOS.

It is generally accepted that PAs themselves are biologically and toxicologically inactive and require metabolic activation. Numerous studies have proven that PAs are first metabolized by CYP3A enzymes in both humans and mice to form DHPAs, which further interact with proteins or DNA and initiate acute and chronic toxicity $[9,32]$. A recent study by Yang et al. [33] proved that senecionine, a highly toxic PA, induced Drp1-mediated mitochondrial fragmentation, which promoted activation of the intrinsic mitochondrial pathway and apoptosis in both primary cultured mouse and human hepatocytes, suggesting that PA-induced mitochondria-mediated hepatocyte apoptosis is conserved from mouse to human. On the other hand, in vitro studies in liver S9 fractions proved that PAs had a shorter half-life in rats than in humans [34]. Detoxification of PAs via the $\mathrm{N}$-glucuronidation reaction catalyzed by UGT1A4 occurs in human liver microsomes but not in liver microsomes of mice and rats, while the rate of activation of PAs via CYP450s is significantly higher in mice and rats than in humans [35]. These findings suggest that mice and rats might be more sensitive to PAs than humans. However, PAinduced HSOS rodent models show similar pathology to human HSOS [36] and have been successfully used to study the functional consequences of PA-induced liver injury [37], as well as in the development of preventative therapeutic strategies [38]. Males are more sensitive to the toxicity of PAs because of the higher metabolic activities of CYP3A and CYP2C11 toward PAs [39, 40]. Thus, male rodents, mainly mice and rats, are usually used to establish PA-induced HSOS models [19-22, 36].

DeLeve et al. developed a reproducible acute HSOS rat model by single oral administration of monocrotaline [36]. In a period of 10 days after treatment, the rats progressed from sinusoidal damage at the earliest stage (day 1 and 2) to late HSOS (day 6 and 7) and severe fibrosis (day 8 and 10). However, monocrotaline promotes the development of pulmonary hypertension in rodents [41], which is not observed in HSOS patients and is caused by exposure to chemotherapy or herbal preparations containing PAs. The presence of such pathology caused by monocrotaline is likely to have a significant physiological impact, thereby limiting the utility of this model in studying either the mechanisms of or therapeutic strategies for HSOS.

Multiple exposures to herbal preparations containing PAs can cause HSOS both in humans and in experimental animals [9, 28]. An investigation was carried out on 56 cases of HSOS secondary to Gynura Rhizoma exposure alone, in which 84 individual patients 
a

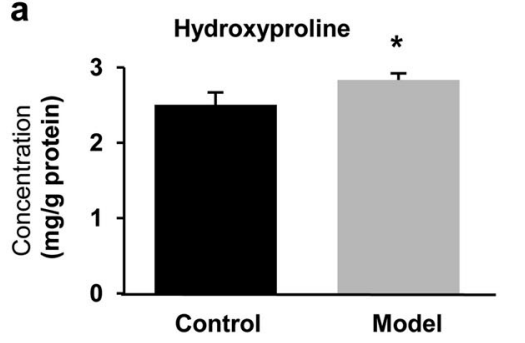

d
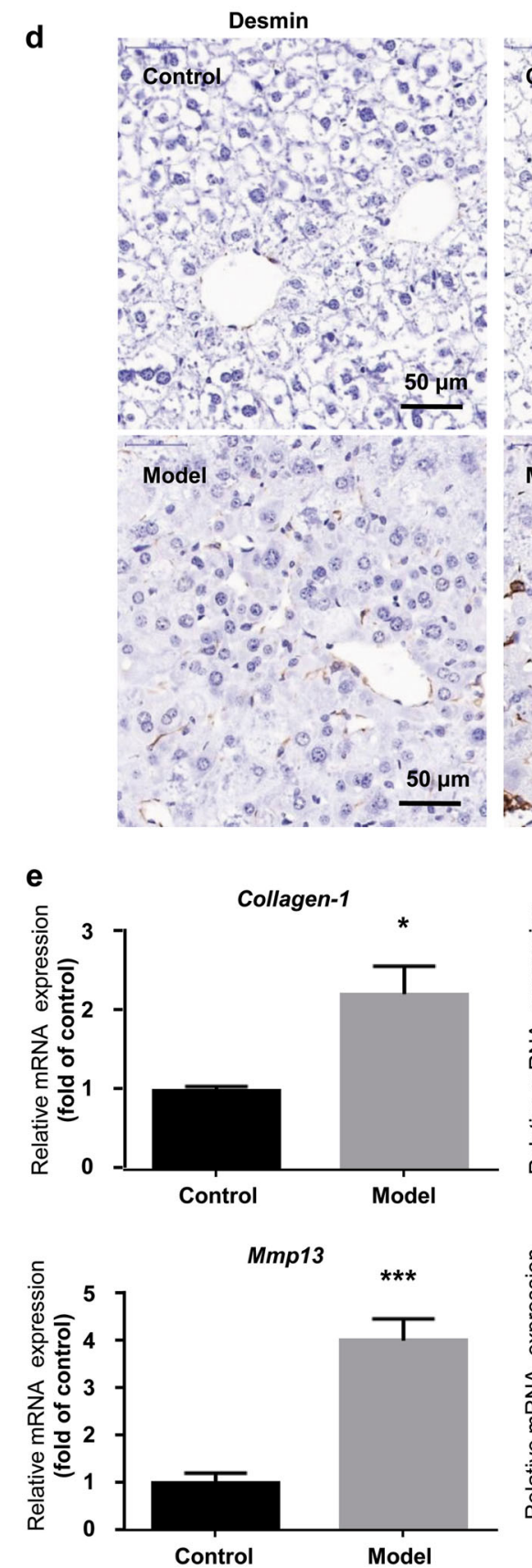

b 드

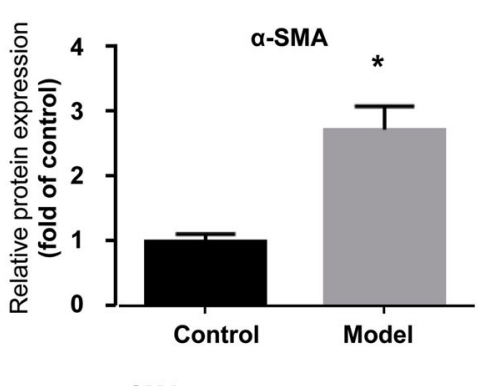

c

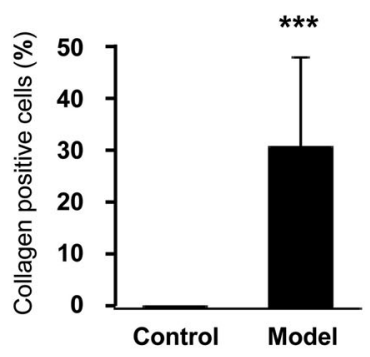

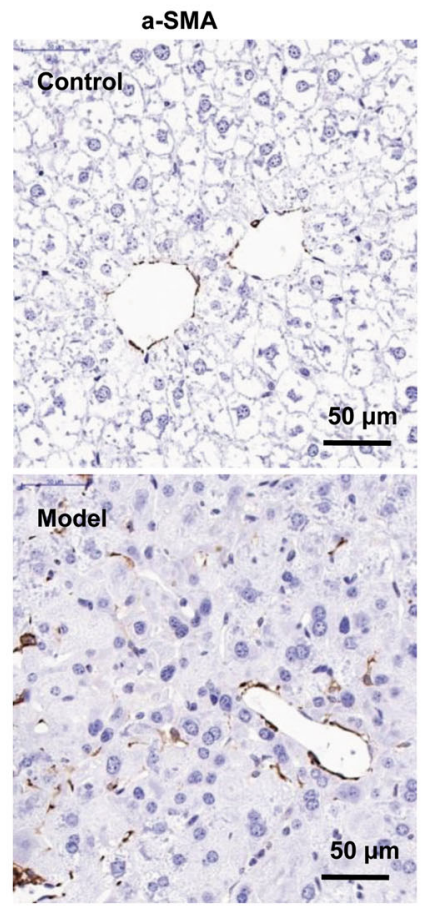
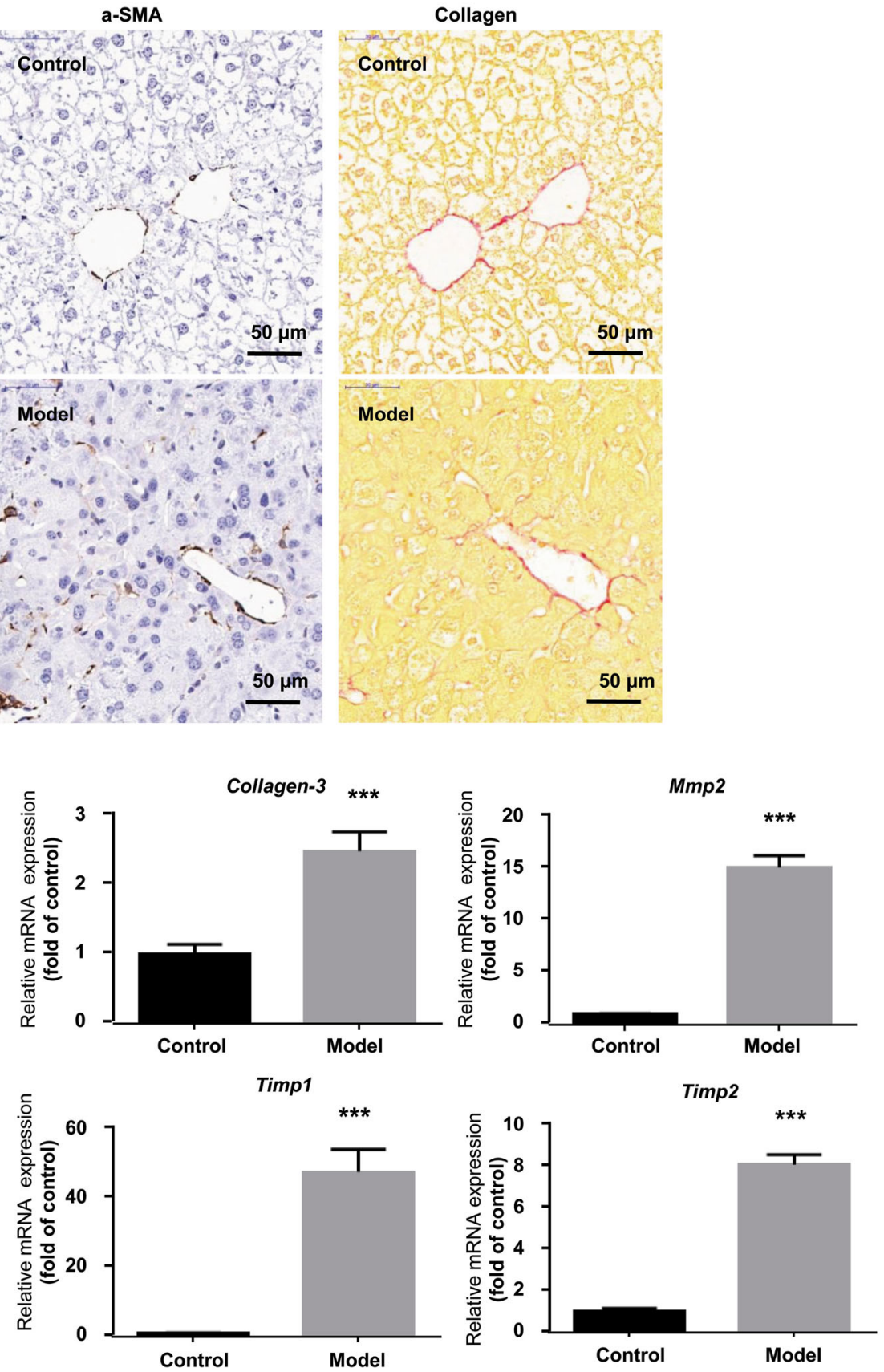

Fig. 2 Evidence of hepatic fibrosis in the HSOS mouse model induced by Gynura Rhizoma. a Hepatic level of hydroxyproline. b Hepatic protein level of $\alpha$-SMA. c Percentage of collagen-positive cells in liver tissue. d IHC staining for desmin and $\alpha$-SMA and Sirus red staining of collagen $(400 \times$ magnification, bar is $50 \mu \mathrm{m})$. Six fields were randomly selected and observed for each sample. e Hepatic mRNA expression of fibrosis-related genes. Values are expressed as the mean \pm SD $(n=6-12)$. Statistics were analyzed by unpaired $t$-test. ${ }^{*} P<0.05,{ }^{* *} P<0.001$ vs. control group 

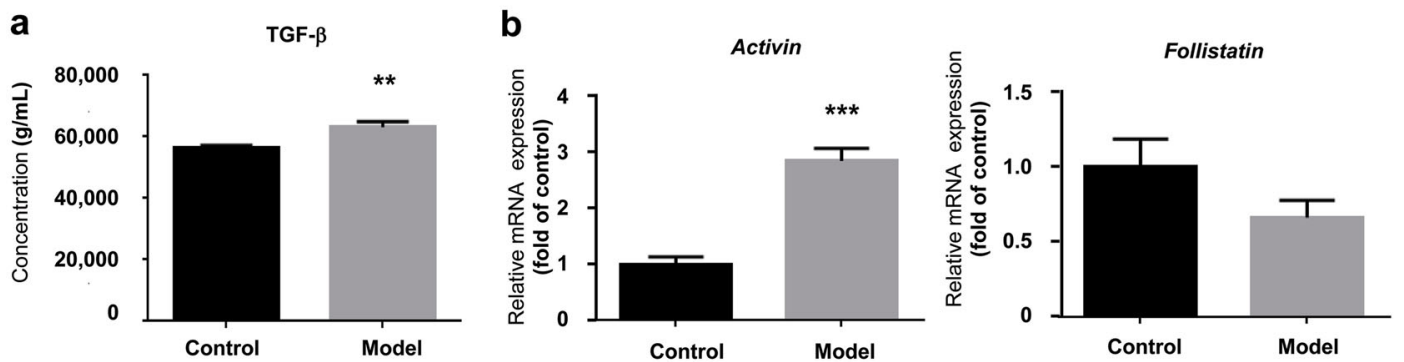

C
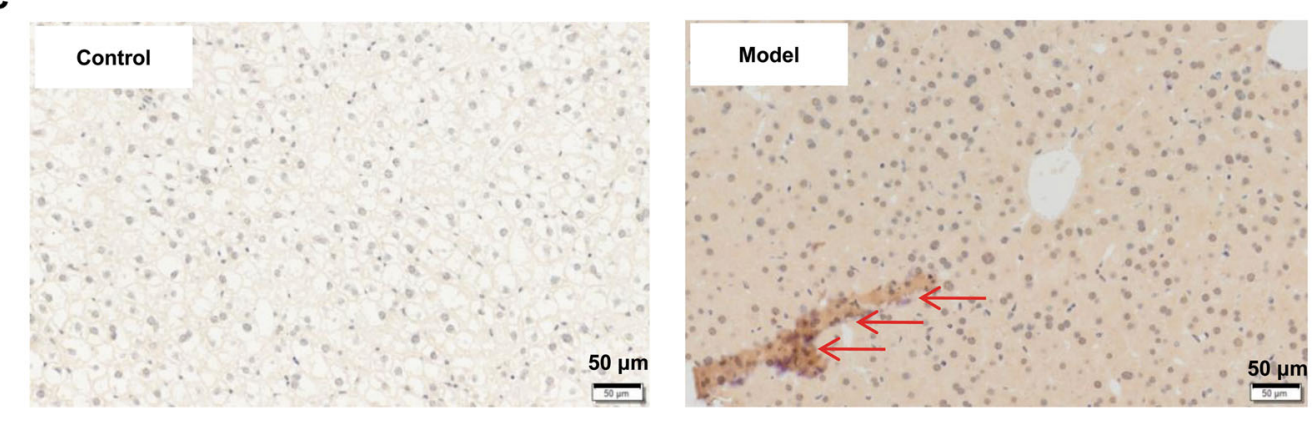

d
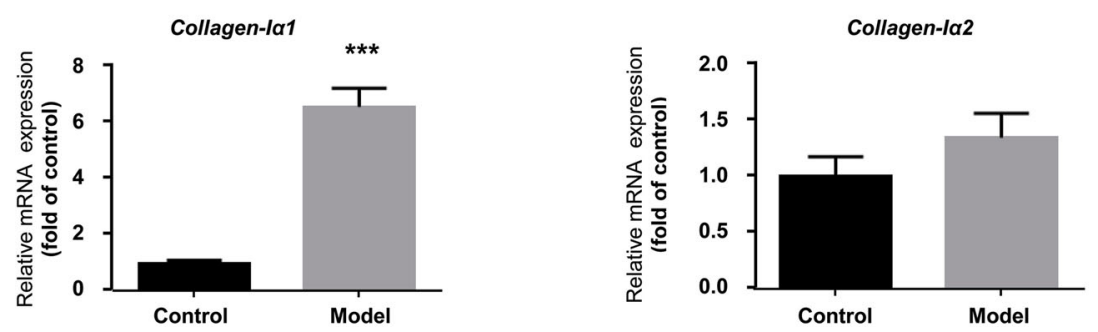

Fig. 3 Key role of TGF- $\beta$-Smad3 signal pathway in the HSOS mouse mode induced by Gynura Rhizoma. a Protein concentration of TGF- $\beta$ in serum. b Hepatic mRNA expression of Activin and Follistatin. c Immunohistochemical staining for p-Smad3 (400x magnification, bar is $50 \mu$ m). The bar represents $50 \mu \mathrm{m}$. Red arrows indicate p-Smad3-positive cells. Six fields were randomly selected and observed for each sample. d Hepatic mRNA expression of Collagen-la1 and Collagen-la2. Values are expressed as the mean \pm SD $(n=6-12)$. Statistics were analyzed by unpaired $t$-test. ${ }^{* *} P<0.01,{ }^{* * *} P<0.001$ vs. control group
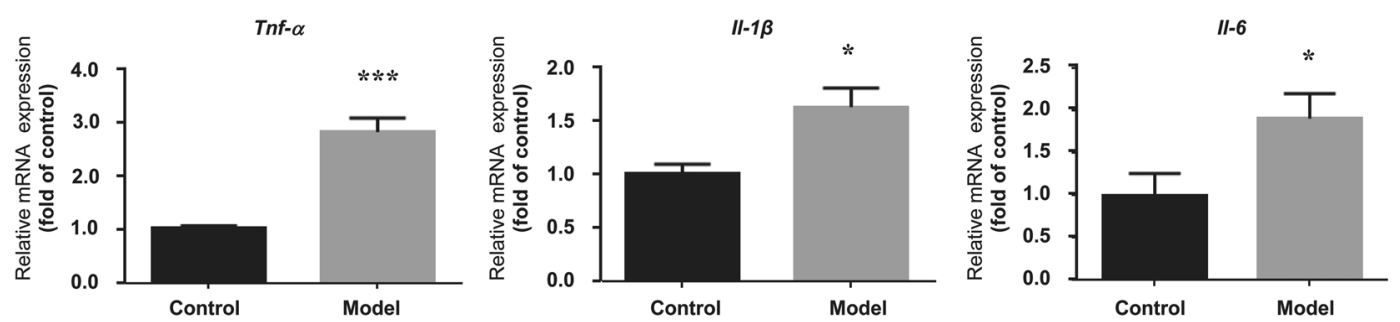

Fig. 4 Hepatic mRNA expression of proinflammatory cytokines. Values are expressed as the mean \pm SD $(n=6)$. Statistics were analyzed by paired $t$-test. ${ }^{*} P<0.05,{ }^{* * *} P<0.001$ vs. control group

were involved. It revealed that the median duration between the use of Gynura Rhizoma and the first onset of clinical symptoms was 1 month (range: 0.2-24 months) [42]. As reported, male rodents are usually treated with $15-30 \mathrm{~g}$ crude drug/kg per day for $3-5$ weeks $[21,22,28]$. However, the contents of toxic compounds, i.e., PAs, were seldom calculated, which made the models hard to reproduce. Therefore, we treated male mice for 40 successive days with standardized Gynura Rhizoma extract to establish the HSOS model in the present study. In this study, the results of serum ALT, AST, and TBIL all indicated Gynura Rhizoma-induced hepatotoxicity in mice. Furthermore, typical pathological changes, including hepatic lobule structure damage, liver cell degeneration and necrosis, and serious fibrosis in the central vein and hepatic sinus, as well as ascites, were consistent with the pathologies of HSOS patients [21, 32]. In contrast, no significant change in HSCs was observed within the sinusoids in any area of the liver lobule in the monocrotaline-induced HSOS model [36]. Furthermore, changes in SEC morphology and MMP9 expression are recognized as the characteristic features of HSOS patients and rodent models [9, 36], and both were observed in our present HSOS mouse model. Therefore, the present model was a typical severe HSOS mouse model.

HSOS begins with endothelial cell damage, with microthromboses and fibrin deposition and causes severe fibrosis in the late stage. A great deal of research has been performed to understand the molecular mechanisms responsible for the development of liver fibrosis [43]. However, limited research has been performed on PA-induced HSOS. Although the activated HSCs are the primary 
cell type responsible for the excess production of collagen during fibrogenesis, some myofibroblast cells, such as portal fibroblasts, can also be activated and contribute to the fibrogenic response [43]. In addition, the activated portal myofibroblasts express classical markers of activated HSCs, including a-SMA. IHC staining for a-SMA and desmin, i.e., the HSC markers, showed colocalization of these two markers in hepatic sinusoids and central veins (Fig. 2d). In addition, a-SMA and desmin-positive cells were significantly increased, suggesting that HSCs were activated and contributed greatly to the fibrogenesis in the HSOS model induced by Gynura Rhizoma.

During fibrogenesis, the release of potent fibrogenic cytokines, such as TGF- $\beta$, plays an essential role. HSCs are a major source of TGF- $\beta$. In addition, TGF- $\beta$ classically engages in intracellular signaling through Smad phosphorylation and regulates downstream gene expression, especially of collagen type I, to promote fibrosis-positive feedback. Our results show that the TGF- $\beta$-Smad signaling pathway was activated. In addition, downstream collagen genes, including Activin and Collagen-la1, were upregulated in the Gynura Rhizoma-induced HSOS model. Clinical and animal studies have shown that activin levels increase in both acute and chronic inflammation. Moreover, inhibition of activin action by its antagonists, such as follistatin, can reduce inflammation, damage, and fibrosis in various disease models. Therefore, the increased hepatic mRNA expression of Activin and the decreased Follistatin suggested severe inflammation and fibrosis in the HSOS mouse model (Fig. 3c). TGF- $\beta$ inhibitors are used for treatment of severe specific pulmonary fibrosis [44], inflammation [45], and myeloma [46] as well. Our study revealed that TGF- $\beta$ inhibitors might also be candidates for the treatment of HSOS.

Zheng et al. [20] reported the activation of inflammatory genes and NF-KB transcriptional activation in an acute HSOS rat model induced by a single treatment with monocrotaline (i.e., $160 \mathrm{mg} /$ $\mathrm{kg})$. Therefore, inflammation should also be associated with the pathogenesis of HSOS. In addition, ingestion of PAs can significantly increase myeloperoxidase activity $[20,47]$, which is also an indicator of inflammation in the liver. Our results demonstrate the existence of inflammation with significantly increased hepatic mRNA expression of Tnf- $\alpha, I I-1 \beta$, and II-6 in the chronic HSOS mouse model induced by multiple administrations of Gynura Rhizoma.

In the present study, we failed to clarify whether inflammation induces early-stage fibrosis or fibrosis induces inflammation. The relationship between fibrosis and inflammation is complicated. As Brown et al. reported [48], a positive feedback loop of inflammation and fibrosis enhances the progression of chronic obstructive pulmonary disease (COPD). In COPD, the intensive response of macrophages, caused by the inhalation of pathogens and particulate, can activate inflammatory cells and pro-inflammatory (TH1) mediators, which results in a feed-forward loop of inflammation, fibrosis, and tissue damage. This feed-forward loop leads to limited airflow and airways with abnormal structure and function, pulmonary vasculature, and lung parenchyma, which are essential features of COPD. In the HSOS mouse model established in present study, this loop may also exist and induce heavy fibrosis. Therefore, blockade of fibrosis or/and inflammation should be an effective treatment of HSOS.

Thus, a severe HSOS mouse model was established in the present study by multiple administrations of Gynura Rhizoma extract, which had well-standardized PA content. The model was validated by typical clinical pathology, including hepatomegaly, ascites, jaundice, elevated serum ALT, AST, and TBIL, and injuries to hepatocytes and SECs, with damaged fenestration and severe congestion. In addition, we revealed that liver fibrosis was induced by triggering the TGF- $\beta$ Smad signaling pathway, and inflammation was strongly induced in the model. Our data proved a feed-forward loop of inflammation, fibrosis, and tissue damage, which suggested a novel treatment strategy for HSOS induced by PA exposure.

\section{ACKNOWLEDGEMENTS}

This work is financially supported by the National Natural Science Foundation (81222053 to Dr. LY and 81603384 to Dr. A-zX), the Shanghai Nature Science Foundation (16ZR1434200 to Dr. A-zX), the Shanghai Rising-Star Program (17QA1403600 to Dr. A-zX), the Program for Shanghai S\&T Achievements Transformation and Industrialization Project (17DZ1920100 to Dr. LY), and the Program of Shanghai Academic/Technology Research Leader (17XD1403500 to Dr. LY).

\section{AUTHOR CONTRIBUTIONS}

The work presented was performed collaboratively by all authors. LY and A-zX defined the research theme and designed the study; $F Z, A-Z X$, and $X Y$ performed the experiments; $F Z, A-Z X$, and $Y Z$ analyzed the data; $L Y$ and $Z-t W$ supervised the study; $F Z, A-Z X$, and $Y Z$ wrote and revised the manuscript. All authors read and approved the final manuscript.

\section{ADDITIONAL INFORMATION}

The online version of this article (https://doi.org/10.1038/s41401-018-0155-y) contains supplementary material, which is available to authorized users.

Competing interests: The authors declare no competing interests.

\section{REFERENCES}

1. Teschke R. Traditional Chinese medicine induced liver injury. J Clin Transl Hepatol. 2014;2:80-94.

2. Teschke R, Wolff A, Frenze C, Schulze J. Review article: herbal hepatotoxicity-an update on traditional Chinese medicine preparations. Aliment Pharmacol Ther. 2014;40:32-50.

3. Frenzel C, Teschke R. Herbal hepatotoxicity: clinical characteristics and listing compilation. Int J Mol Sci. 2016;17:588.

4. Danan G, Teschke R. RUCAM in drug and herb induced liver injury: the update. Int J Mol Sci. 2016;17:14.

5. Teschke R, Larrey D, Melchart D, Danan G. Traditional Chinese Medicine (TCM) and herbal hepatotoxicity: RUCAM and the role of novel diagnostic biomarkers such as microRNAs. Medicines. 2016;3:18.

6. WHO. International Programme on Chemical Safety, International Programme on Chemical Safety. Pyrrolizidine alkaloids health and safety guide; health and safety guide no. 26. Geneva: WHO; 1989.

7. Chen MY, Cai JT, Du Q. Hepatic veno-occlusive disease associated with the use of Gynura segetum. Eur J Intern Med. 2007;18:609-16.

8. Dai N, Yu YC, Ren TH, Wu JG, Jiang Y, Shen LG, et al. Gynura root induces hepatic veno-occlusive disease: a case report and review of the literature. World J Gastroenterol. 2007;13:1628-31.

9. Lin G, Wang JY, Li N, Li M, Gao H, Ji Y, et al. Hepatic sinusoidal obstruction syndrome associated with consumption of Gynura segetum. J Hepatol. 2011;54:666-73.

10. Deleve LD, Mccuskey RS, Wang XD, Hu LP, Mccuskey MK, Epstein RB, et al. Medicinal plants in China containing pyrrolizidine alkaloids. Pharmazie. 2000;55:711-26.

11. Stegelmeier BL, Edgar JA, Colegate SM, Gardner DR, Schoch TK. Pyrrolizidine alkaloid plants, metabolism and toxicity. J Nat Toxins. 1999;8:95-116.

12. Yang MB, Ruan JQ, Fu PP, Lin G. Cytotoxicity of pyrrolizidine alkaloid in human hepatic parenchymal and sinusoidal endothelial cells: firm evidence for the reactive metabolites mediated pyrrolizidine alkaloid-induced hepatotoxicity. Chem Biol Interact. 2016;243:119-26.

13. Gao H, Li N, Wang JY, Zhang SC, Lin G. Definitive diagnosis of hepatic sinusoidal obstruction syndrome induced by pyrrolizidine alkaloids. J Dig Dis. 2012;13:33-9.

14. Huang ZL, Chen M, Zhang J, Sheng Y, Ji LL. Integrative analysis of hepatic microRNA and mRNA to identify potential biological pathways associated with monocrotaline-induced liver injury in mice. Toxicol Appl Pharmacol. 2017;333:35-42.

15. DeLeve LD, Ito Y, Machen NW, McCuskey MK, Wang XD, McCuskey RS. Embolization by sinusoidal lining cells causes the congestion of hepatic veno occlusive disease. Gastroenterology. 2000;118:A1003.

16. Shulman HM, Fisher LB, Schoch HG, Kenne KW, McDonald GB. Veno-occlusive disease of the liver after marrow transplantation: histological correlates of clinical signs and symptoms. Hepatology. 1994;19:1171-80.

17. Watanabe $K$, Iwaki $H$, Satoh $M$, Ikeda $T$, Ichimiya $S$, Suzuki $N$, et al. Veno-occlusive disease of the liver following bone marrow transplantation: a clinical-pathological study of autopsy cases. Artif Organs. 1996;20:1145-50. 
18. Keating GM. Defibrotide: a review of its use in severe hepatic veno-occlusive disease following haematopoietic stem cell transplantation. Clin Drug Invest. 2014;34:895-904.

19. Zhang J, Sheng Y, Shi L, Zheng Z, Chen M, Lu B, et al. Quercetin and baicalein suppress monocrotaline-induced hepatic sinusoidal obstruction syndrome in rats. Eur J Pharmacol. 2017;795:160-8.

20. Zheng Z, Shi L, Sheng Y, Zhang J, Lu B, Ji LL. Chlorogenic acid suppresses monocrotaline-induced sinusoidal obstruction syndrome: the potential contribution of NFkB, Egr1, Nrf2, MAPKs and PI3K signals. Environ Toxicol Pharmacol. 2016;46:80-9.

21. Chen Z, Huo JR, Yang L, Zhu HY. Effect of ligustrazine on mouse model of hepatic veno-occlusive disease induced by Gynura segetum. J Gastro Hepatol. 2011;26:1016-21.

22. Zhu HY, Chu Y, Huo JR, Chen Z, Yang L. Effect of prednisone on transforming growth factor- $\beta 1$, connective tissue growth factor, nuclear factor-kBp65 and tumor necrosis factor- $\beta$ expression in a murine model of hepatic sinusoidal obstruction syndrome induced by Gynura segetum. Hepatol Res. 2011;41:795-803.

23. Fang LX, Xiong AZ, Yang X, Cheng WZ, Yang L, Wang ZT. Mass-spectrometrydirected analysis and purification of pyrrolizidine alkaloid Cis/trans isomers in Gynura japonica. J Sep Sci. 2014;37:2032-8.

24. Yang M, Ruan J, Gao H, Li N, Ma J, Xue J, et al. First evidence of pyrrolizidine alkaloid $\mathrm{N}$-oxide-induced hepatic sinusoidal obstruction syndrome in humans. Arch Toxicol. 2017;91:3913-25.

25. Helmy A. Review article: updates in the pathogenesis and therapy of hepatic sinusoidal obstruction syndrome. Aliment Pharmacol Ther. 2006;23:11-25.

26. Wadleigh M, Ho V, Momtaz P, Richardson P. Hepatic veno-occlusive disease: pathogenesis, diagnosis and treatment. Curr Opin Fematol. 2003;10:451-62.

27. Nakamura K, Hatano E, Narita M, Hayashino AM, Koyama Y, Nagata $Y$, et al. Sorafenib attenuates monocrotaline-induced sinusoidal obstruction syndrome in rats through suppression of JNK and MMP-9. J Hepatol. 2012;57j:1037-43.

28. Yu XZ, Ji T, Bai XJ, Liang L, Wang LY, Chen W, et al. Expression of MMP-9 in hepatic sinusoidal obstruction syndrome induced by Gynura segetum. J Zhejiang Univ-Sci B (Biomed \& Biotechnol). 2013;14:68-75.

29. Miguel AM-M, María JT-L, Aarón FG-C, Belinda V-C. A capillary electrophoresis method for the determination of hydroxyproline as a collagen content index in meat products. Food Anal Methods. 2012;5:464-70.

30. Wu LM, Wu XX, Sun Y, Kong XW, Zhang YH, Xu Q. A novel synthetic oleanolic acid derivative (CPU-II2) attenuates liver fibrosis in mice through regulating the function of hepatic stellate cells. J Biomed Sci. 2008;15:251-9.

31. Robinson SM, Mann J, Vasilaki A, Mathers J, Burt AD, Oakley F, et al. Pathogenesis of FOLFOX induced sinusoidal obstruction syndrome in a murine chemotherapy model. J Hepatol. 2013;59:318-26.

32. Zhu L, Xue J, Xia Q, Fu PP, Lin G. The long persistence of pyrrolizidine alkaloidderived DNA adducts in vivo: kinetic study following single and multiple exposures in male ICR mice. Arch Toxicol. 2017;91:949-65.
33. Yang $X$, Wang $H$, Ni HM, Xiong AZ, Wang ZT, Sesaki $H$, et al. Inhibition of Drp1 protects against senecionine-induced mitochondriamediated apoptosis in primary hepatocytes and in mice. Redox Biol. 2017;12:264-73.

34. Kolrep F, Numata J, Kneuer C, Preiss-Weigert A, Lahrssen-Wiederholt M, Schrenk $D$, et al. In vitro biotransformation of pyrrolizidine alkaloids in different species. Part I: microsomal degradation. Arch Toxicol. 2018;92:1089-97.

35. He YQ, Yang L, Liu HX, Zhang JW, Liu Y, Fong A, et al. Glucuronidation, a new metabolic pathway for pyrrolizidine alkaloids. Chem Res Toxicol. 2010;23: 591-9.

36. DeLeve LD, McCuskey RS, Wang X, Hu L, McCuskey MK, Epstein RB, et al. Characterization of a reproducible rat model of hepatic veno-occlusive disease. Hepatology. 1999;29:1779-91.

37. DeLeve LD, Wang X, Kanel GC, Ito Y, Bethea NW, McCuskey MK, et al. Decreased hepatic nitric oxide production contributes to the development of rat sinusoidal obstruction syndrome. Hepatology. 2003;38:900-8.

38. Deleve LD, Wang X, Tsai J, Kanel G, Strasberg S, Tokes ZA. Sinusoidal obstruction syndrome (veno-occlusive disease) in the rat is prevented by matrix metalloproteinase inhibition. Gastroenterology. 2003;125:882-90.

39. Chung WG, Buhler DR. The effect of spironolactone treatment on the cytochrome P450-mediated metabolism of the pyrrolizidine alkaloid senecionine by hepatic microsomes from rats and guinea pigs. Toxicol Appl Pharmacol. 1994;127: 314-9.

40. Lin G, Cui YY, Liu XQ. Gender differences in microsomal metabolic activation of hepatotoxic clivorine in rat. Chem Res Toxicol. 2003;16:768-74.

41. Schultze $A E$, Roth RA. Chronic pulmonary hypertension-the monocrotaline model and involvement of the hemostatic system. J Toxicol Environ Health B Crit Rev. 1998;1:271-346.

42. Wang XX, Qi XS, Guo XZ. Tusanqi-related sinusoidal obstruction syndrome in China: a systematic review of the literatures. Medicine. 2015;94:e942.

43. Tsukada S, Parsons CJ, Rippe RA. Mechanisms of liver fibrosis. Clin Chim Acta. 2006;364:33-60.

44. Shao MX, Wang WT, Zhao ZY, Tang LD. A new drug treating idiopathic pulmonary fibrosis: pirfenidone. Drugs Clin. 2013;28:409-14.

45. Wan X, Li ZG, Ying JM. Effect of transforming growth factor beta (TGF- $\beta$ ) receptor I kinase inhibitor on prostate cancer bone growth. Bone. 2012;50: 695-703.

46. Bueno $L$, Alwis DP, Pitou C. Semi-mechanistic modeling of the tumor growth inhibitory effects of LY2157299, a new type I receptor TGF- $\beta$ kinase antagonist, in mice. Eur J Cancer. 2008;44:142-50.

47. Ahmed LA, Obaid AA, Zaki HF, Agha AM. Role of oxidative stress, inflammation, nitric oxide and transforming growth factor-beta in the protective effect of diosgenin in monocrotaline-induced pulmonary hypertension in rats. Eur Pharmacol. 2014;740:379-87.

48. Brown BN, Price IM, Toapanta FR. An agent-based model of inflammation and fibrosis following particulate exposure in the lung. Math Biosci. 2011;231: 186-96. 